\title{
The Effects of High Definition Transcranial Direct Current Stimulation Targeting the Primary Motor Cortex on Experimental Capsaicin-Induced Pain in Healthy Volunteers
}

\author{
SAEID METWALLY, M.Sc. ${ }^{\mathbf{1}}$; HASSAN KOTB, M.D. ${ }^{1}$; ALAA ATTIA, M.D. ${ }_{\mathbf{5}}{ }^{\text {; }}$ EMAN KHEDR, M.D. ${ }^{\mathbf{2}}$; \\ SAM CHONG, Ph.D. ${ }^{3}$; FREDRIC BOY, Ph.D. ${ }^{4}$ and ROMAN CREGG, Ph.D. \\ The Departments of Anesthesia \& Pain Management and Neurology ${ }^{2}$, Faculty of Medicine, Assiut University, Assiut, Egypt, \\ The Department of Neurology and Pain Management, National Hospital for Neurology and Neurosurgery, London, UK ${ }^{3}$, \\ The Department of Psychology, School of Management, Swansea University, Swansea, UK ${ }^{4}$ and \\ The Department of Anesthesia and Pain Management, National Hospital for Neurology and Neurosurgery, London, UK ${ }^{5}$
}

\begin{abstract}
Background: Chronic pain is one of the most common health problems in the world and a significant challenge to clinical practice. Which has severe effects on patient's social and daily work that costs a lot of the governmental support. Traditionally, the focus on alleviating chronic pain has relied mainly on pharmacotherapy which has limited efficacy and significant side-effects, especially with of prolonged use. Especially with opioids which is the most widely prescribed drugs for pain.

Transcranial Direct Current Stimulation (tDCS) which deliver a weak current up to $(2 \mathrm{~mA})$ through electrodes on the scalp has attracted much interest as it is safe, inexpensive and easy to implement and can be used as an alternative or adjuvant to pharmacotherapy.
\end{abstract}

Aim of Study: In this study, we aimed to test the effects of the newly developed and more focally targeted anodal High Definition transcranial Direct Current Stimulation (HD-tCDS) targeting the PMC on a capsaicin-induced pain model in healthy subjects. We set out to test the hypothesis that it is possible to use HD-tDCS to stimulate the PMC and to compare this with sham stimulation.

Patients and Methods: After obtaining informed consent, 36 healthy volunteers (20 male). All volunteers subjected to the following experimental paradigm: Application of capsaicin cream $(0.075 \%)$ on a $9 \mathrm{~cm}^{2}$ area of skin for 30 minutes on the volar surface of both forearms. The volunteers were blinded and then divided into two groups of 18 with each subjected to 20 minutes of the following: Group 1 received Sham HD tDCS targeting PMC, group 2 anodal HD tDCS (2mA) targets the PMC. Pain severity was collected using Numerical Rating Scale (NRS) score at regular intervals before and after the session. The subjects also asked whether they were able to differentiate between sham and active stimulation and any complications of stimulation recorded. The NRS scores also compared between the two groups. Statistical significance

Correspondence to: Dr. Saeid Metwally, The Department of Anesthesia \& Pain Management, Faculty of Medicine, Assiut University Hospital, Assiut, Egypt, was calculated using suitable tests and $p<0.05$ accepted as the level of significance.

Results: Compared to sham stimulation, the PMC stimulation by HD-tDCS associated with a faster reduction in NRS pain scores after application of capsaicin to forearm skin. Also, it was well tolerated without documented adverse effect that necessitates discontinuation of the session.

Conclusion: Anodal HD-tDCS of the primary motor cortex was associated with a faster reduction in reported pain score when compared with sham stimulation. Which can be used as a new treatment option for different chronic pain conditions.

Key Words: Capsaicin-induced pain - Primary motor cortex - High Definition Transcranial Direct Current Stimulatioon (HD tDCS).

\section{Introduction}

PAIN is a significant health problem all over the world with an incidence of $30 \%$ of the population had experienced chronic pain, and $8.2-8.9 \%$ of them experienced neuropathic pain, with severe effects on their social and daily work that costs a lot of the governmental support [1].

Pain in the brain concept; is an attractive subject and means that the pain matrix is activated whenever the brain concludes that the body tissue is in danger and action is necessary. This supported by the use of imaging techniques where the Magnetoencephalography (MEG), functional MRI (fMRI) and Positron Emission Tomography (PET) are the leading imaging methods which revealed six crucial mechanisms including cortical reorganisation, maladaptive plasticity, increased primary nociceptive areas, recruitment of new cortical regions, modified endogenous pain modulation and neurochemistry changes [2] 
Advanced imaging studies revealed neuroanatomical correlates of pain including spinal cord, brain stem and thalamus as well as various parts of the limbic system such as the hypothalamus, amygdala, hippocampus, anterior cingulate cortex, insular cortex, somatosensory cortex, motor cortex and prefrontal cortex [3]

Neuropathic pain is associated with the reorganisation of cortical sensory maps as pain leads to disruption of functional connectivity which can sometimes be restored by effective treatment. Because of the neural circuity of the brain operates via electrostimulation, applying a small current to the scalp has been investigated as a means of modulating brain activity [4].

The International Neuromodulation Society defines therapeutic neuromodulation as "the alteration of nerve activity through targeted delivery of a stimulus, such as electrical stimulation or chemical agents, to specific neurological sites in the body [5]. In appropriate patients, this growing class of therapies can help restore function or relieve symptoms that have a neurological basis.

The reversible therapy delivers stimulation to specific neural circuits in the brain, spine, or peripheral nerves. Depending on the target, the therapy may be non-invasive or minimally invasive. Neuromodulation approaches range from noninvasive techniques such as transcranial electric stimulation to implanted devices, such as spinal cord stimulation or a deep brain stimulation.

Despite the advent of new drugs and therapeutic regimens, a significant proportion of chronic pain patients remain symptomatic despite best pharmacological treatment. Several lines of research have looked for new strategies to control refractory pain, among which Non-Invasive Brain Stimulation (NIBS) techniques. In recent years, these techniques were mainly represented by repetitive Transcranial Magnetic Stimulation (rTMS) and transcranial Direct Current Stimulation (tDCS), which based on different mechanisms of action [6].

In transmagnetic stimulation, the magnetic field penetrates the scalp and skull and induces a small current parallel to the plane of the coil in the brain. When the induced current is sufficient, neuronal membranes depolarise and action potentials are generated. While transcranial Direct Current Stimulation (tDCS) which deliver a weak current up to $(2 \mathrm{~mA})$ through electrodes on the scalp has attracted much interest as it is safe, inexpensive and easy to implement [7].
There are two common types of transcranial Direct Current Stimulation (tDCS), the first one is the conventional (tDCS) As direct current stimulation conveys, aweak current that activates relatively large brain areas most of the current applied is shunted because of the high impedance of the scalp and skull dispersion. One of the main disadvantages of the conventional tDCS is the diffuse effect and lack of focality [8]

Recently, Datta et al., [9] proposed that the second type which called High Definition transcranial Direct Current (HD tDCS) can results in more significant brain modulation with enhanced spatial focality compared to conventional tDCS. HighDefinition transcranial Direct Current Stimulation (HD-tDCS) has been recently introduced to improve the spatial accuracy of conventional. By using arrays of smaller "high definition" electrodes, instead of the two large pad electrodes $[9,10]$.

A diminished electrode size has been shown to reduce affected cortical area size and therefore increase focality. Targeting a brain area using HDtDCS is achieved by placing the electrodes in a predetermined configuration to rationally guide current flow. HD-tDCS can be provided using a variety of montages having different positions and a different number of electrodes [11]

There are two types of stimulation anodal (increase the excitability of the brain cortex) and cathodal (which decrease the cortical excitability), also anodal stimulation means the electric current is going from the active electrode to the other electrode while the reverse occur in cathodal stimulation where the current is going from negative electrode to the active one [8].

In the early 1990s, Tsubokawa and colleagues [12] presented that epidural motor cortex stimulation was effective in treating thalamic pain syndromes, it has been recently stated that over 700 patients were treated with motor cortex stimulation worldwide with a variety of protocols, because of this heterogeneity comparison of the results remain difficult [13].

Activation of the various inter-neural circuit within the primary motor cortex, inducing antidromic modulation of the thalamocortical fibres with remote activation of different brain regions especially insula, cingulate cortex, thalamus and Periaqueductal Grey (PAG) [14]

Also, motor cortex stimulation is known to enhance release of endogenous opioids in painrelated circuits as the PAG, and cingulate cortex, 
the link between PMC stimulation and N-MethylD-Aspartate (NMDA) receptors can explain its long-lasting analgesic effect [13].

Human experimental pain models can act as a translational bridge between animal and clinical research, and many of the mechanisms tested in animals can also be translated and evaluated in healthy volunteers and used to predict the efficacy of a given drug in specific patient populations. Capsaicin pain model can produce neuropathy like pattern response in the form of pain, allodynia and hyperalgesia which is a standard presentation in neuropathic pain.

Cutaneous application of capsaicin-induced tonic pain associated with neuropathic like pattern of pain, hyperalgesia and allodynia which mimic symptoms in classical neuropathic pain patients [15].

Although our understanding of pain mechanisms has significantly progressed through the development of neuro-techniques, experimental tools are still essential and widely used. Among these necessary experimental tools for the study of mechanisms and development of new analgesics, capsaicin pain model is considered as one of the most important sources of knowledge in the pain field. Many recent studies reported that capsaicin could also be used to relieve pain. This paradox can also seen with opioids which have an established clinical use as analgesics but also induce hyperalgesia [16]

Where capsaicin is a compound found in chilli peppers and responsible for their burning and irritant effect. In addition to heat sensation, capsaicin produces pain and for this reason is an essential tool in the study of pain. Capsaicin induces painlike behaviour by activation of TRPV1 receptors expressed by nociceptors. The seminal work of Szolcsanyi [17] demonstrates that capsaicin selectively acts on C-polymodal nociceptors and the thermodependency of sensory effects on animals and humans. The interaction between the capsaicin and TRPV1 receptors change the selectivity to inwards ions as increases the influx of calcium, and the activity of different kinases such as PKA and $\mathrm{PKC}$ which contributes to the higher perception of pain [18].

\section{Patients and Methods}

\section{Participants:}

The study was conducted at the Center for Health and Environmental Management Research \& Innovation, Swansea University in direct collaboration with University College London, between
October 2016 to December 2017. A total of 36 healthy volunteers (20 male, 16 females, mean age 31.27 years and range 20-55 years) gave informed written consent to take part in the study. All participants were able to provide informed consent, follow the study protocol and withdraw from the study at any point. The study performed in agreement with the Helsinki Declaration and the protocol reviewed by the local ethics committee of Swansea University.

\section{Inclusion criteria:}

Able to give informed consent to participate in the study, male and female University undergraduate, postgraduate and staff and ASA I to II participants.

\section{Exclusion criteria:}

A participant who had any history of, an adverse reaction to TMS/tDCS, seizure, an unexplained loss of consciousness, a stroke, serious head injury, surgery on their head, any brain related, neurological illnesses, any illness that may have caused brain injury, frequent or severe headaches, metal in the head (outside the mouth) such as shrapnel, surgical clips, or fragments from welding, any implanted medical devices such as cardiac pacemaker's or medical pumps, taking any analgesic medications in the past 24 hours, pregnancy and if anyone in his family has epilepsy.

\section{Withdrawal criteria:}

Participant request or participant unable to complete the session for any reasons.

\section{The general design of the study:}

36 healthy volunteers who fulfil the inclusion criteria randomly assigned to two groups of 18 participants each.

A- Group 1 received Sham HD-tDCS targeting the Primary Motor Cortex (PMC).

B- Group 2 received active (anodal) HD-tDCS $(2 \mathrm{~mA})$ targeting the PMC.

Sample size:

Sample size calculation based on a review of previous studies using a capsaicin pain model, we determined that such sample size was sufficient and able to generate robust data potentially. For instance, in two recent capsaicin-induced pain neuromodulation studies, researchers used either 14 healthy participants Sacco et al., [19] or 16 participants in Fierro et al., [20]. Alternatively, we determined the necessary sample size through a power calculation using $\mathrm{G}$ power 3. 1.9.3 software (Dusseldorf, Germany). An a priori estimation 
indicated a sample size of 34 patients divided into two groups $(n=17)$ to detect a clinically-relevant $1.5 \mathrm{~cm}$ reduction (average standard deviation $1.5 \mathrm{~cm}$ ) in pain VAS level intensity at $p<.025$ with a power of $80 \%$. To account for the multiple outcomes and dropouts, we increased the sample size to 18 per group.

\section{Randomization:}

Participants were consecutively assigned to a randomisation scheme generated on the website Randomization.com (Dallal GE, http://www. randomization.com, 2008). We used the second generator, with random permutations for a 3-group trial. The randomisation sequence was concealed until interventions were assigned. Generation of the random allocation sequence and assignment of participants were performed by an independent investigator not involved in the selection or assessment of the volunteers.

\section{Blinding:}

To prevent the introduction of bias, all participants and the trained investigator who performed the assessments were blind to the type of stimulation. Once baseline assessments had been conducted, a different researcher was allowed to check the randomisation code for the volunteer. He then set up the montage, operated the device, and delivered the stimulation accordingly. The assessor was not present in the room during delivery of the stimulation. Once the HD-tDCS session had ended, and the operator removed the equipment, the assessor came back to the room and finished the assessments. The participants were blinded to the type of intervention.

\section{Outcome measures:}

\section{Primary outcome measures:}

- Evaluation of whether focal HD-tDCS over the motor cortex could decrease pain intensity and sensory experience among healthy adult volunteers using Numerical Rating Score (NRS) from (0-100) immediately after capsaicin application and every 5 minutes after that (for 30 minutes in total), and every 5 minutes during the applications of the HD-tDCS session (for 20 minutes in total).

\section{Secondary outcome measures:}

- Safety and tolerability of HD-tDCS among volunteers.

\section{The setting of the study:}

The experiment conducted in a quiet room with an average temperature of $25^{\circ} \mathrm{C}$ with volunteers sitting in a comfortable chair. All participants read an information sheet about the research before completing a screening questionnaire for HDtDCS, and finally filling in a consent form, and after the HD tDCS session, they fill exit questionnaire.

\section{The procedure:}

After skin preparation (alcoholic gel), a $3 \mathrm{~cm}$ $X 3 \mathrm{~cm}$ square area was defined on the dried volar surface of each forearm at about $6 \mathrm{~cm}$ from the elbow crease. The investigator applied about 1.5 grams of $0.075 \%$ capsaicin cream in each area. The spread of capsaicin cream outside the area of application prevented by using surgical tape. After 30-mn, the capsaicin cream and the adhesive tape removed. An area of erythema observed at the site of cream application and the surrounding zone.

During the 30 minutes of capsaicin cream application, the pain was subjectively assessed by using Numerical Rating Scale (NRS 1-100) Fig. (1) at the time of cream application and every 5 minutes throughout the 30 minutes of cream application.

This subjective tool for pain assessment (NRS) where (0) means no pain while (100) is the worst possible pain.

After the baseline assessment of pain scores volunteers subjected to 20 minutes of either sham or active HD-tDCS (randomised allocation). Side effects and changes in NRS recorded during the HD-tDCS session every 5 minutes, as well as the time participant started feeling improvement (i.e., initial pain reduction).

\section{$H D$-tDCS equipment and session:}

HD-tDCS was administered with a current intensity of $2 \mathrm{~mA}$ for $20 \mathrm{~min}$ (anodal HD-tDCS) by employing a $4 \times 1$ multichannel adaptor (CBL204, Biopac System Inc., Goleta, CA, USA) connected to a conventional tDCS battery-driven constant current stimulator (NeuroConn $\mathrm{GmbH}$, Ilmenau, Germany). Stimulation was delivered using silver/silver chloride sintered ring electrodes (ELTP-RNG Sintered; Stens Biofeedback Inc., San Rafael, CA, USA). Electrodes held in place by specially designed plastic casings embedded in modular Electroencephalography (EEG) recording cap. Fig. (2A,B,C).

We positioned the centre electrode (anode) according to study group over $\mathrm{C} 3$ (for motor cortex stimulation) based on the international 10/20 EEG System, which corresponds approximately to the location of the left M1. Four return electrodes (cathode) placed in a radius of about $7.5 \mathrm{~cm}$ from 
the centre electrode. Their positions corresponded roughly to CZ, F3, T7, and P3 Fig. (3).

The hair underlying each electrode separated to expose the scalp, and approximately $1.5 \mathrm{ml}$ of highly conductive gel (Sigma Gel; Parker Laboratories, Fairfield, NJ) was placed beneath each electrode to improve conductance. During each active 4 X 1 HD-tDCS session; DC was gradually ramped up over a period of 30 s until reaching an intensity of $2 \mathrm{~mA}$, which delivered for 20 minutes for the active (anodal) stimulation. These parameters have previously been shown to be well tolerated in healthy subjects.

The same montage was used for the sham procedure; however, the current was applied for 30 seconds only. This sham tDCS stimulation has been used and shown not to alter cortical excitability.

Fig. (1): Numerical rating scale used as pain assessment tool.

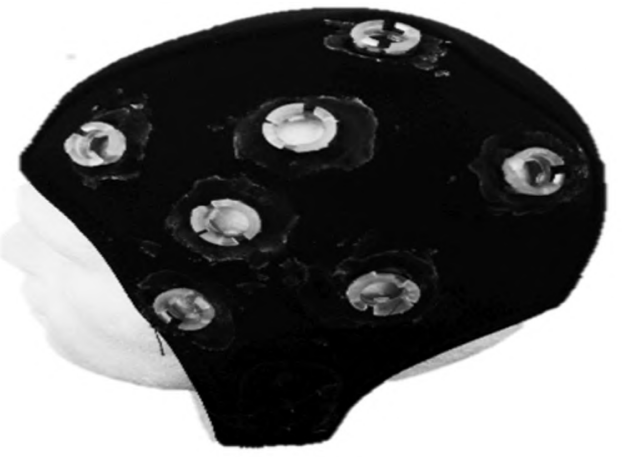

(A)

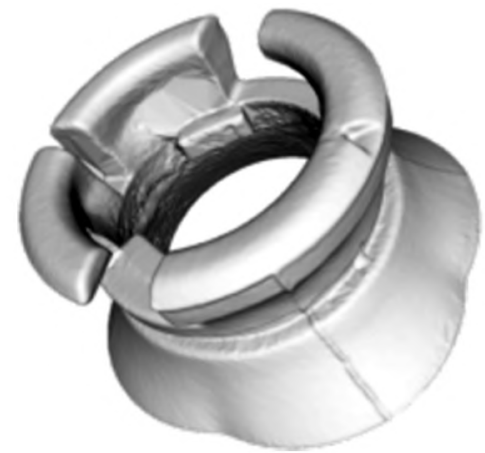

(B)

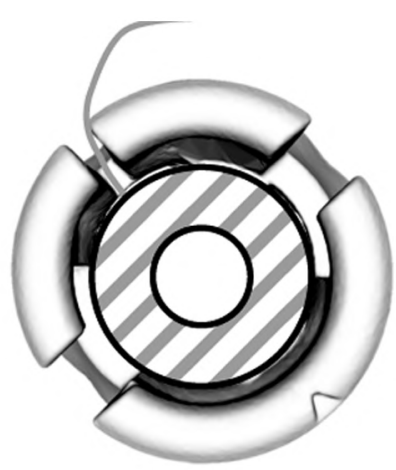

(C)

Fig. (2): Cap and plastic casing used in HD tDCS session: (A) The recording cap with the embedded plastic casing. (B\&C) Shows models of the designed plastic casing

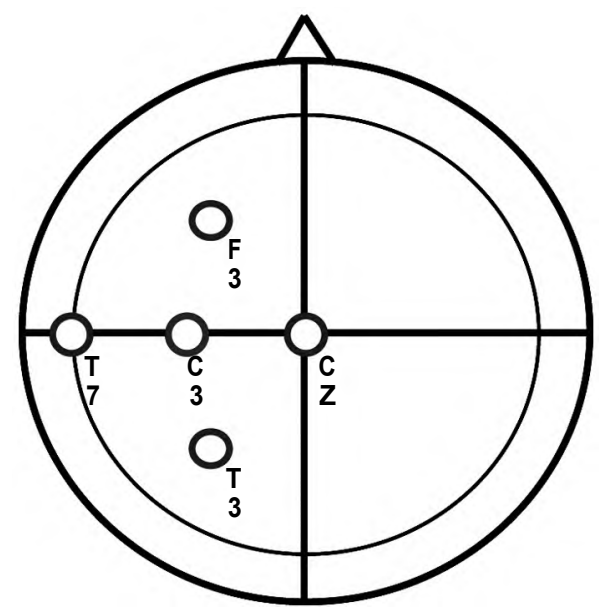

Fig. (3): The selected montage for brain stimulation with HD tDCS. The montaging used for motor cortex stimulation.

\section{Statistical analysis:}

Data were analysed using SPSS, Version 22.0 (SPSS Inc., Chicago, IL, USA) software programs. The data were tested for normality using the Shapiro-Wilks test and for homogeneity variances before further statistical analysis and expressed as the mean \pm standard error of the mean unless stated otherwise; error bars in the figures represent standard errors. Categorical variables described by number and proportion $(\mathrm{N}, \%)$.

The ratings of pain intensity were obtained from all subjects (NRS) in two different conditions; the real HD tDCS session targeting the primary motor cortex (motor group) and the sham stimulation session (sham group). The time courses of the rating were compared with the two-way factorial analysis of variance (ANOVA) with repeated measurements (condition and time) followed by posthoc analysis with Protected Least Significant Difference (PLSD) for multiple comparisons were used to assess the interaction between groups at different points of assessment. A $p$-value of less than 0.05 was considered statistically significant.

Two-way ANOVA was used to compare the time course of capsaicin-induced pain with Condition (two levels: Motor and sham HD tDCS) and 
time (twelve levels: $0,5,10,15,20,30$, at start of the HD tDCS session, 5min during session, 10min during session, $15 \mathrm{~min}$ during the session and $20 \mathrm{~min}$ at the end of the session) as within-subject factors.

To analyse carry over effect one way repeated measures ANOVA used for the baseline values of the dependent variables.

The Greenhouse-Geissner correction of degrees of freedom was used when necessary to correct for non-sphericity of the data.

A chi-square test was used to evaluate the proportion of volunteers correctly guessing whether they had received real or sham HD-tDCS.

\section{Results}

Performance and tolerability of the HD tDCS stimulation:

All 36 volunteers tolerated the procedures well, and few minor adverse effects were reported (Table 1). A tingling sensation was perceived by all subjects at the onset of stimulation. This sensation was continuously felt for longer than two min from the start of active stimulation by 11 volunteers in the active motor group. Three participants in the PMC stimulation group experienced a burning sensation at the site of the active electrode. Sleepiness was reported by 4 participants in the motor groups. An itching sensation at the location of the anodal electrode was reported one participant in the PMC group. One participant in the PMC group reported a headache during the session.

Assessment of pain score before and after $H D$ tDCS session:

At the time of capsaicin cream application, participant's ratings on the subjective pain Numerical Rating Scale (pNRS) were not different (all subjective ratings at zero). As expected, there is a significant increase in pain ratings as time passes, after active motor HD-tDCS stimulation; we noticed that subjective pain ratings rapidly decreased during the session which is close to zero Fig. (4).

One way repeated measure ANOVA revealed that the baseline values of dependent variables (pain perception and type of stimulation) remained unchanged in multiple session for all conditions of stimulation, this excludes the possibility of carry-over effect from the previous stimulus on the same healthy volunteer. There was no statistically significant difference found from capsaicin application to its removal (i.e., the moment at which HD-tDCS started) Fig. (4).

Table (1): Frequency of side effects across the study groups. Numbers and proportion in (\%) of reported side effects across the study groups.

\begin{tabular}{llccccc}
\hline $\begin{array}{l}\text { Side effects/ } \\
\text { stimulation type }\end{array}$ & $\begin{array}{c}\text { Tingling } \\
\text { sensation }\end{array}$ & $\begin{array}{c}\text { Burning } \\
\text { sensation }\end{array}$ & $\begin{array}{c}\text { Itching } \\
\text { sensation }\end{array}$ & Sleepiness & A headache & $\begin{array}{c}\text { Acute mood } \\
\text { changes }\end{array}$ \\
\hline Sham & $5(27 \%)$ & 0 & 0 & 0 & 0 & 0 \\
Motor & $11(61.1 \%)$ & $3(16.66)$ & $1(5.55 \%)$ & $4(22.22 \%)$ & $1(5.55 \%)$ & 0 \\
\hline
\end{tabular}

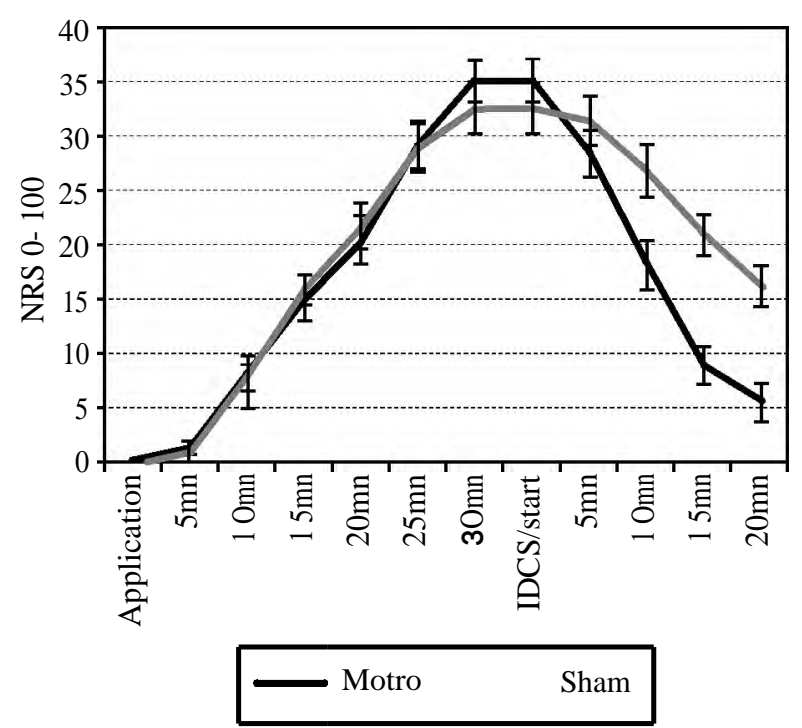

Fig. (4): Changes in NRS in relation to time.
The graph shows the time courses of pain intensity rating between the study groups. Mean scores of pain intensity ( - \pm SEM) at every 5 minutes in the study groups are shown (blue, sham group; orange, motor group). A two-way factorial ANOVA revealed significant effects of both condition and time. A significant interaction was also found between them. Posthoc $t$-test analysis showed that at $10-20 \mathrm{~min}$, the rating in the active conditions were significantly lower than those in the sham condition.

\section{Minimum duration to start feeling initial pain} reduction:

When comparing the minimal duration of HDtDCS stimulation to feel an initial improvement in subjective pain score across the study groups, We note that it took less time for the participant 
to start feeling an improvement in the active group as opposed to the sham condition (motor vs sham: $t(17)=4.534, p=0.00014$ ) (Table 2). It takes about $4 \mathrm{~min}$ in the active groups and about $10 \mathrm{~min}$ in the sham group Fig. (5).

Table (2): Mean and SEM of minimum duration in minutes to start feeling initial pain reduction across the study groups.

\begin{tabular}{lll}
\hline & Motor & Sham \\
\hline Mean (min) & 4.94 & 10.56 \\
SEM & 0.47 & 1.14 \\
\hline
\end{tabular}

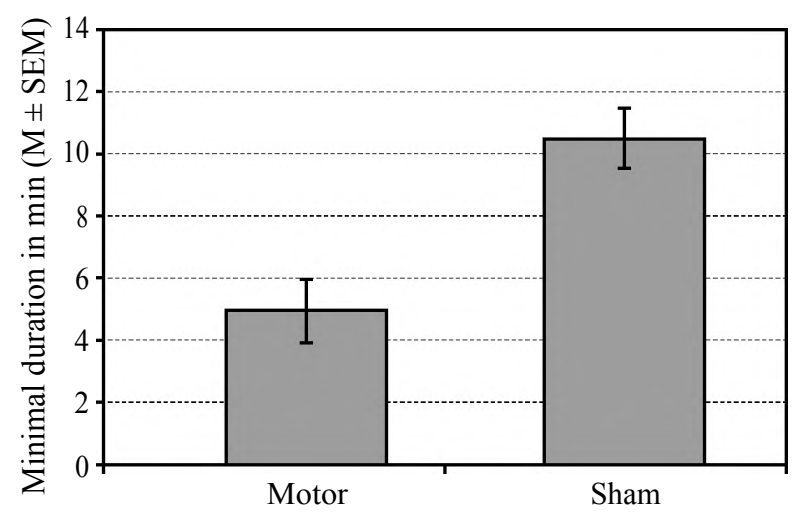

Fig. (5): Minimal duration of HD-tDCS stimulation to start feeling an initial improvement in subjective pain score NRS (mean value \pm SEM).

The validity of the blinding technique with sham stimulation:

The results of the chi-square tests with respect to the blinding of the study showed that subjects were able to correctly guess above chance whether they received real or sham stimulation in the active stimulation group compared to sham group, $p=$ 0.014 which support the alternative hypothesis that stated the participants can correctly guess the type of stimulation and do not support the null hypothesis which stated the participants could not correctly guess the type of stimulation they received.

\section{Discussion}

This study has been designed to assess the effect of HD tDCS on pain perception and sensory changes associated with classical experimental topical capsaicin pain model in healthy volunteers, selecting the PMC as the primary target area of stimulation. Which may confirm its efficacy for clinical use the main findings were:

1- Active stimulation was tolerable and didn't generate significant adverse effects.

2- Capsaicin cream application can be a suitable experimental model for producing neuropathic like pattern that produces some of the critical features of neuropathic pain like pain, hyperalgesia and allodynia.

3- Active stimulation targeting the motor cortex relative to sham stimulation decreases the pain score resulted from capsaicin application.

Participants in the current study didn't report significant adverse effects induced by the HD tDCS. This finding in agreement with other studies used HD tDCS stimulation and found no significant adverse effects as in Borchardt et al., [21], Xu et al., [22] and Wing et al., [23] .

In this study, participants were able to correctly guess above chance whether they received real or sham stimulation in the active stimulation groups compared to sham group. Our result showing that $77.8 \%$ of the real groups are correctly guessing the type of stimulation in comparison with $44.4 \%$ in the sham group correctly guess the type of stimulation. While Wing et al., [23] their results shows that $57 \%$ of the participants were able to guess what condition they received (real or sham HD tDCS) and Borchardt et al., [21] their results showed that $46 \%$ in the real group correctly guess the type of stimulation in comparison with $18 \%$ in the sham group.

We found a significant reduction in subjective pain scores (NRS) and areas of primary and secondary hyperalgesia after motor cortex HD-tCDS compared to sham stimulation. We found that volunteers in the stimulation groups also reported a significantly faster reduction in pain scores compared to the sham group.

Little work has been done by NIBS modality in combination with the capsaicin pain model. These studies using different modalities of brain stimulation either rTMS or tES with different outcomes.

In this trial as regard subjective pain assessments our results show that at the time of capsaicin cream application, participant's ratings on the subjective pain Numerical Rating Scale (pNRS) were not different (all subjective ratings at zero). As expected there is a significant increase in pain ratings as time passes, after active motor and insular cortex stimulation we noticed that subjective pain ratings rapidly decreased during the session which is close to zero again.

Our experimental pain model shows the same time course as presented in Harding et al., [24] as it takes 5-10 minutes to produce pain, with the peak at 30 minutes, which succeeded to create mild 
to moderate pain. In Harding et al., [24] onset of burning/tingling sensation typically developed approximately $5 \mathrm{~min}$ after application. The intensity of sensation then increased to reach a maximum by $20 \mathrm{~min}$ after application. There was no statistical difference in evoked sensation at 20,30 and 40min.

Concerning the time courses of pain rating in the study groups after the applications of HD tDCS stimulation. A two-way factorial ANOVA revealed that there was a statistically significant effect of both condition and time. A significant interaction was also found between the two factors. The posthoc analysis showed a statistically significant differences between the motor cortex stimulation versus the sham group at each point from 10 to 20min of the HD tDCS stimulation when the rating was significantly lower in the active stimulation conditions. These results suggest that HD tDCS produced an earlier, more rapid reduction of pain over the motor cortex than the sham stimulation in acute pain elicited by capsaicin cream application.

We are not aware of any other studies that investigated the effect of HD-tDCS on the capsaicin model of pain. Some studies have used rTMS (Tamura et al., [25], Fierro et al., [20], Sacco et al., [19]). These studies have reported that rTMS to the contralateral PMC (Tamura et al., [25] and left DLPFC (Fierro et al., [20] reported reduced VAS pain scores in volunteers subjected to application of topical capsaicin.

In the study by Sacco and colleagues, [19] they used pain scores as well as quantitative sensory testing in a combined heat/capsaicin pain model. They reported that rTMS to the PMC is superior to stimulation of the DLPFC or midline occipital cortex in reducing pain scores after application of capsaicin.

The benefits of rTMS stimulation of the PMC was also supported by the study of Tamura et al., (2004) [25] . In an attempt to clarify the action mechanism of rTMS, another study measured MEP (Motor Evoked Potentials) in a topical capsaicin model (Fierro et al., 2010) [20]. Consistent with other studies, they also reported that rTMS to the DLPFC reduces pain. They further showed that pain after capsaicin application decreases corticospinal pathway activation with reduced MEP amplitude and lowers intra-cortical inhibition. These changes were reversed by rTMS to the DLPFC.

Although relatively few studies have been published on HD-tDCS so far, it has been shown to reliably target specific brain areas and has shown to produce plastic changes that may outlast more than conventional tDCS as suggested by Kuo et al., [26]

In agreement with Boggio et al., [27] who tested the modulatory effects of anodal transcranial direct current stimulation on pain perception and pain thresholds in healthy volunteers. They tested it on twenty healthy subjects received stimulation with tDCS under four different conditions of stimulation: anodal tDCS of the motor cortex, DLPFC, occipital cortex and sham tDCS. Their results ultimately suggest that M1 stimulation produces an analgesic effect by modulating the sensory aspects of pain, while DLPFC stimulation mediates its effects by modulating affective-emotional networks associated with pain, especially unpleasantness associated with pain.

Also, there is an interesting study carried by Borckardt and their colleagues in (2012) [21] in which they are preliminarily testing the safety and tolerability of the HD-tDCS technique as well as to evaluate whether HD-tDCS over the motor cortex would decrease pain and sensory experience. Their results revealed that HD-tDCS appears well tolerated, and produced changes in underlying cortex that are associated with changes in pain perception.

All the studies mentioned above supported the results of this study that showed that pain perception could be improved and modulated using HD tDCS targeting the PMC.

Although tDCS treatment has been used to treat different pathological conditions, its mechanism has not been fully elucidated yet. Animal and cellular model studies demonstrated that tDCS is capable of modulating synaptic transmission, molecular biosynthesis, neuronal morphology, and different neurotransmitters' system [28] .

DosSantos et al., [29]. Has shown that a single session of anodal tDCS over the motor cortex results in a reduction of mu opioid receptor binding of an exogenous receptor ligand in the pain matrix, suggesting that the analgesic effect of M1-tDCS may be due to a direct increase of endogenous opioid release. The authors suggest that the decreased binding of the exogenous ligand was possibly due to receptor occupancy by enhanced release of endogenous opioids. The reduction was detected in numerous cortical and subcortical structures of the pain matrix, such as nucleus accumbens, anterior cingulate cortex, insula and thalamus, and was accompanied by an increased threshold for experimentally induced cold pain. Although opioid analgesic effects are known to relate to both the 
emotional as well as the sensory dimension of pain, no significant changes in clinical pain levels were elicited after a single tDCS session. Suggesting that the immediate opioidergic effects of a single tDCS application are subclinical, and repeated application might be necessary to get clinically meaningful results.

Another possibility that the active place boresponse cant ruled out perhaps direct current can be an active placebo, and the difference between patients is dependent on their level of expectation.

\section{Limitations:}

1- Blinding is not perfect as subjects were still more likely to be able to tell the difference between the active and sham stimulation.

2- Another limitation is that we tested a capsaicin model by applying capsaicin cream with low concentration $(0.075 \%)$ so the stability of the model is not guaranteed for a long time, and this may explain the reduction in pain score in the sham group, so we need a more stable pain model.

3- Although quite big by other standards, this is still a small study: Only 18 volunteers in each group.

4- Objective neuroimaging measures were necessary to confirm the changes occurs as a result of the stimulation and the possible underlying mechanisms.

\section{Conclusion:}

Anodal stimulation of HD-tDCS targeting the primary motor cortex was associated with a faster reduction in reported pain score in a capsaicin induced model of experimental pain when compared with sham stimulation. Also the stimulation was safe well tolerable without major adverse effects which open the door for clinical application of the more focally targetd HD tDCS.

\section{References}

1- SMITH B.H., TORRANCE N., BENNETT M.I. and LEE A.J.: Health and quality of life associated with chronic pain of predominantly neuropathic origin in the community. The Clinical Journal of Pain, 23 (2): 143-9, 2007.

2- SATOR-KATZENSCHLAGER S.: Pain and neuroplasticity. Revista Médica Clínica Las Condes, 25 (4): 699 706, 2014.

3- APKARIAN A.V., BUSHNELL M.C., TREEDE R.D. and ZUBIETA J.K.: Human brain mechanisms of pain perception and regulation in health and disease. Eur. J. Pain, 9: 463-84 [PubMed], 2005.

4- Medical University of South Carolina. (2018, March 27). Detection of transcranial direct current stimulation deep in the living human brain. ScienceDaily. Retrieved April 20, 2018 from www.sciencedaily.com/releases/2018/03/ 180327162610.htm.

5- International Neuromodulation Society. Accessed Dec. 21, 2016. Welcome to the International Neuromodulation Society. www.neuromodulation.com

6- MYLIUS V., BORCKARDT J.J. and LEFAUCHEUR J.P.: Noninvasive cortical modulation of experimental pain. Pain, 153 (7): pp. 1350-63, 2012.

7- THAIR H., HOLLOWAY A.L., NEWPORT R. and SMITH A.D.: Transcranial Direct Current Stimulation (tDCS): A Beginner's Guide for Design and Implementation. Frontiers in Neuroscience, 11, 2017.

8- MARSTON L., SPENCER S., DeSOUZA L.H. and WAND B.M.: Non-invasive brain stimulation techniques for chronic pain, 2018

9- DATTA A., BANSAL V., DIAZ J., PATEL J., REATO D. and BIKSON M.: Gyri-precise head model of transcranial direct current stimulation: Improved spatial focality using a ring electrode versus conventional rectangular pad. Brain Stimul, 2 (4): 201-7, 207 e201** compares the focality of conventional tDCS with HD-tDCS, 2009.

10- VILLAMAR M.F., VOLZ M.S., BIKSON M., DATTA A., DaSILVA A.F. and FREGNI F.: Technique and considerations in the use of $4 \times 1$ ring high-definition transcranial direct current stimulation (HD-tDCS). Journal of visualized experiments: JoVE, (77), 2013.

11- DMOCHOWSKI J.P., DATTA A., BIKSON M., SU Y. and PARRA L.C.: Optimized multi-electrode stimulation increases focality and intensity at the target. Journal of Neural Engineering, 8 (4): p. 046011, 2011.

12- TSUBOKAWA T., YAMAMOTO T., KATAYAMA Y., HIRAYAMA T., MAEJIMA S. and MORIYA T.: Deepbrain stimulation in a persistent vegetative state: Followup results and criteria for selection of candidates. Brain Injury, 4 (4): 315-27, 1990.

13- HENSSEN D.J., KURT E., VAN WALSUM A.M.V.C., ARNTS I., DOORDUIN J., KOZICZ T. and BARTELS R.H.: Long-term effect of motor cortex stimulation in patients suffering from chronic neuropathic pain: An observational study. PloS one, 13 (1): e0191774 13333 , 2018.

14-DosSANTOS M.F., FERREIRA N., TOBACK R.L., CARVALHO A.C. and DaSILVA A.F.: Potential mechanisms supporting the value of motor cortex stimulation to treat chronic pain syndromes. Frontiers in Neuroscience, 10, $18,2016$.

15- KUMAR REDDY K.S., NAIDU M.U.R., RANI P.U. and RAO T.R.K.: Human experimental pain models: A review of standardized methods in drug development. Journal of Research in Medical Sciences: The Official Journal of Isfahan University of Medical Sciences, 17 (6): p. 587, 2012.

16- FATTORI V., HOHMANN M.S., ROSSANEIS A.C., PINHO-RIBEIRO F.A. and VERRI W.A.: Capsaicin: Current understanding of its mechanisms and therapy of pain and other pre-clinical and clinical uses. Molecules, 21 (7): 844, 2016. 
17- SZOLCSANYI J.: A pharmacological approach to elucidation of the role of different nerve fibres and receptor endings in mediation of pain. J. Physiol., 73: 251-9, 1977.

18-YANG F., XIAO X., CHENG W., YANG W., YU P., SONG Z., YAROV-YAROVOY V. and ZHENG J.: Structural mechanismunderlying capsaicin binding and activation of the TRPV1 ion channel. Nat. Chem. Biol., 11: 518-24. [CrossRef] [PubMed], 2015.

19- SACCO P., PRIOR M., POOLE H. and NURMIKKO T.: Repetitive transcranial magnetic stimulation over primary motor vs non-motor cortical targets; effects on experimental hyperalgesia in healthy subjects. B.M.C. Neurology, Sep. 4, 14 (1): 166, 2014.

20- FIERRO B., De TOMMASO M., GIGLIA F., GIGLIA G., PALERMO A. and BRIGHINA F.: Repetitive transcranial magnetic stimulation (rTMS) of the dorsolateral prefrontal cortex (DLPFC) during capsaicin-induced pain: Modulatory effects on motor cortex excitability. Exp. Brain Res., 203: 31-8, 2010.

21- BORCKARDT J.J., BIKSON M., FROHMAN H., REEVES S.T., DATTA A., BANSAL V., et al.: A pilot study of the tolerability and effects of high-definition transcranial direct current stimulation (HD-tDCS) on pain perception. J. Pain, 13 (2): 112-20, 2012.

22- XU J., HEALY S.M., TRUONG D.Q., DATTA A., BIKSON M. and POTENZA M.N.: Focus: Addiction: A Feasibility Study of Bilateral Anodal Stimulation of the Prefrontal Cortex Using High-Definition Electrodes in Healthy Participants. The Yale Journal of Biology and Medicine. Sep., 88 (3): 219, 2015.

23- WING T. , EROH J., HART J. and VANNESTE S.: Exploring the effects of anodal and cathodal high definition transcranial direct current stimulation targeting the dorsal anterior cingulate cortex. Scientific reports, 8 (1): 4454 , 2018.

24- HARDING L.M., MURPHY A., KINNMAN E. and BARANOWSKI A.P.: Characterization of secondary hyperalgesia produced by topical capsaicin jelly-a new experimental tool for pain research. European Journal of Pain, 5 (4): 363-71, 2001.

25- TAMURA Y., OKABE S., OHNISHI T. , N. SAITO D. ARAI N., MOCHIO S., INOUE K. and UGAWA Y.: Effects of 1-Hz repetitive transcranial magnetic stimulation on acute pain induced by capsaicin. Pain, 107 (1-2): $107-$ $15,2004$.

26- KUO H.I., BIKSON M., DATTA A., MINHAS P., PAULUS W., KUO M.F., et al.: Comparing cortical plasticity induced by conventional and high-definition $4 \mathrm{X} 1 \mathrm{ring}$ tDCS: A neurophysiological study. Brain Stimul., 6: 6448, 2013.

27- BOGGIO P.S., ZAGHI S., LOPES M. and FREGNI F.: Modulatory effects of anodal transcranial direct current stimulation on perception and pain thresholds in healthy volunteers. European Journal of Neurology, 15 (10): 112430, 2008.

28- MEDEIROS L.F., De SOUZA I.C., VIDOR L.P., et al.: Neurobiological effects of transcranial direct current stimulation: A review. Frontiers in psychiatry, 3, 110 * review of neurobiological effects of tDCS, 2012.

29- DosSANTOS M.F., LOVE T.M., MARTIKAINEN I.K., NASCIMENTO T.D., FREGNI F., CUMMIFORD C., et al.: Immediate effects of tDCS on the mu-opioid system of a chronic patient. Front. Psychiatry, 3: 93 10.3389/ fpsyt.2012.00093 [PMC free article] [PubMed] [Cross Ref], 2012. 


\section{مقارنة بين تآثير تحفيز المغ الغير إختراقى العالى الدقة للقشرة الحركية الآولية

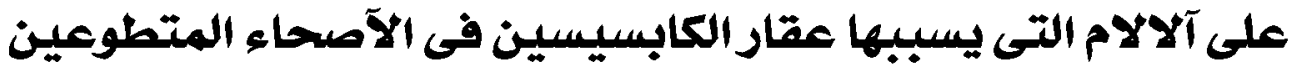

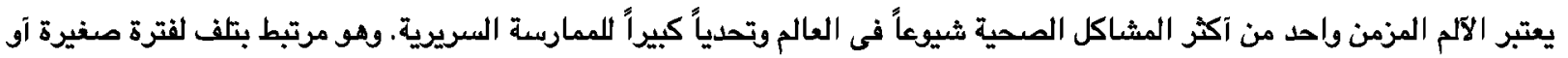

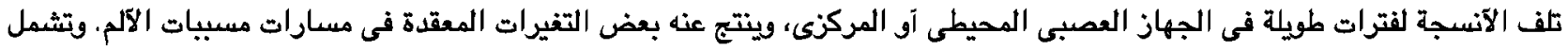

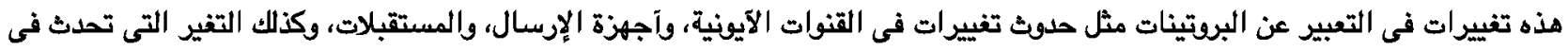
الدوائر العصبية من الجهاز العصبير عنى المرتينات المركنى.

ويمثل الإنتشار المرتفع للآلم المزمن العالمى ونسبة حدوثه المرتفعه، والآمراض المصاحبة لحدوثه، وإرتباطه بالعديد من المحددات الإجتماعية

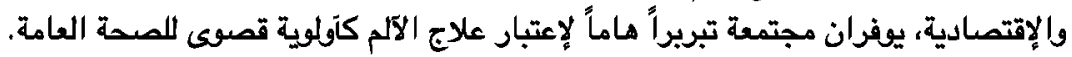

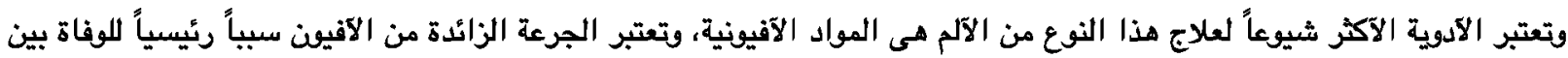

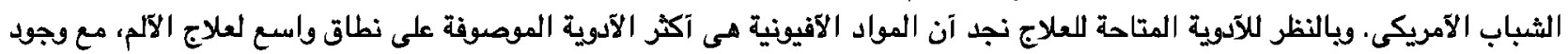

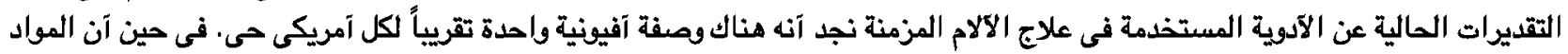

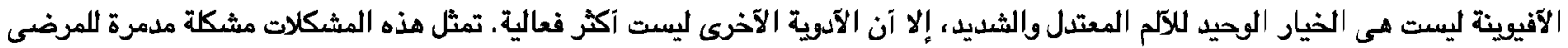
وآنظمة الرعاية الصحية والمجتمعية.

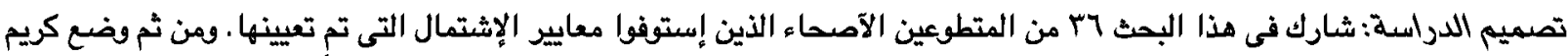

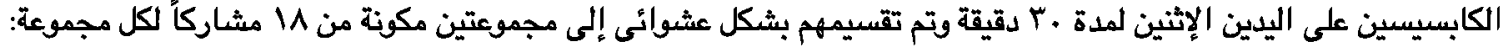

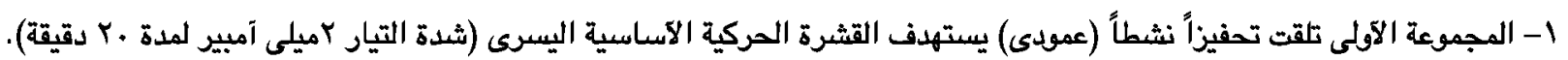

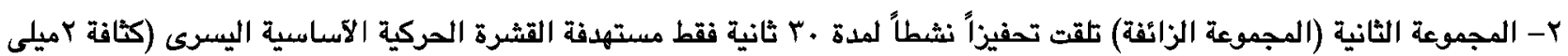
لمدة .ب ثانية فقط ثم عادت إلى الصفر ثم إستكملت مدة . ب دقيقة). ץ- التحفيز النشط الذى إستهدف القشرة الحركية (PMC) نسبة إلى التحفيز الشام يقلل من درجة الآلم الناتجة عن وضع الكابسيسين كريم.

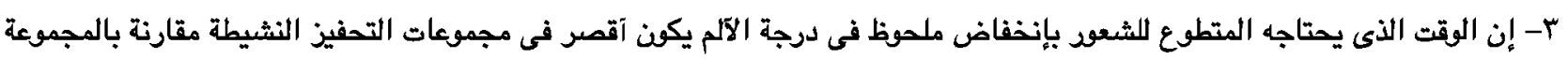
الزائفة.

ع- يمكن تحفيز المخ الغير إختراقى عالى الدقة فى الطريقة التى قمنا بها هذا الحد من التصسيس الآولية والثانوية آكثر من التحفيز الزائغة

محددات هذه الدراسة:

ا- التعمية ليست مثالية حيث كان من المرجح آكثر آن تكنن قادرة على معرفة الفرق بين التحفيز النشط والشامل.

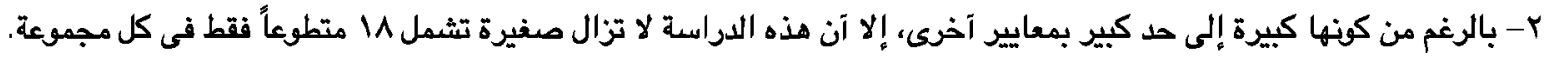
r- كانت تدابير التصوير العصبى الموضوعية ضصوية لتاكيد حدوث التغيرات كتيجة للتحفيز والآليات الآساسية المحتملة. ع- التاتثير عابر والذى يمكن آن يعزى إلى التحفيز لجلسة واحدة، لإختبار تآثير طويل الآمد من التحفيز HD tDCS يمكن القيام به مع جلسات 